\title{
OVERVIEW OF THE SCIENTIFIC OBJECTIVES OF THE HIGH CURRENT EXPERIMENT FOR HEAVY-ION FUSION*
}

\author{
P. Seidl', R. Bangerter, C. Celata, A. Faltens, V. Karpenko, E. Lee, LBNL, Berkeley CA 94720, I. Haber, NRL, \\ Washington, D.C. 20375, S. Lund, A. Molvik, LLNL, Livermore, CA 94550
}

\begin{abstract}
The High Current Experiment (HCX) is being built to explore heavy-ion beam transport at a scale appropriate to the low-energy end of a driver for fusion energy production. The primary mission of this experiment is to investigate aperture fill factors acceptable for the transport of space-charge dominated heavy-ion beams at high space-charge intensity (line-charge density $\sim 0.2 \mu \mathrm{C} / \mathrm{m}$ ) over long pulse durations $(3-10 \mu \mathrm{sec})$. A single beam transport channel will be used to evaluate scientific and technological issues resulting from the transport of an intense beam subject to applied field nonlinearities, envelope mismatch, misalignment-induced centroid excursions, imperfect vacuum, halo, background gas and electron effects resulting from lost beam ions. Emphasis will be on the influence of these effects on beam control and limiting degradations in beam quality (emittance growth). Electrostatic (Phase I) and magnetic (Phase II) quadrupole focusing lattices have been designed and future phases of the experiment may involve acceleration and/or pulse compression. The Phase I lattice is presently under construction[1] and simulations to better predict machine performance are being carried out [2]. Here we overview: the scientific objectives of the overall project, processes that will be explored, and transport lattices developed.
\end{abstract}

\section{INTRODUCTION}

The Heavy-Ion-Fusion (HIF) Program's objective is to provide a comprehensive scientific knowledge base for inertial fusion energy (IFE) driven by high-brightness heavy-ion beams. The HCX will explore beam transport limitations at a scale characteristic of the low-energy end of a multi-beam induction linac driver, using a single beam to save on cost. Advances in the understanding of the physics of intense ion beam transport and acceleration of a single beam will enable better determination of the optimal beam aperture filling factor and pulse duration. Filling the apertures as much as possible is very important in multi-beam induction linacs in order to minimize the transverse cross-sectional area of multi-beam focusing arrays and, in turn, the amount of induction core material needed for economical acceleration. Thus, it is important to maximize the beam current density over the crosssection of the array. The optimum will be linked to the choice of beam parameters and focusing technology. Small beam-edge to aperture clearances at high intensities

\footnotetext{
* This work supported by the Office of Energy Research, U.S. Department of Energy, the Lawrence Berkeley and Livermore Labs under contract number DE-AC03-76SF00098 and W-7405-Eng-48. $†$ PASeidl@lbl.gov
}

make it crucial to understand how processes influence practical transport limits. These limits may be different in magnetic and electric focusing sections, and most costoptimal driver concepts employ both electric (low energy) and magnetic (higher energy) focusing sections.

The single-beam HCX will be the first heavy-ion electric and magnetic quadrupole transport experiment with simultaneously: high line charge density $(\lambda \sim 0.2$ $\mu \mathrm{C} / \mathrm{m})$, long pulse duration $(3<\tau<10 \mu \mathrm{s})$, and high injection energy $\left(1.6<\mathrm{E}<2.0 \mathrm{MeV}, \mathrm{K}^{+}\right.$, coasting beam). These parameters, for a driver, are believed to lie in the rather broad ranges: $0.1<\lambda<1.0 \mu \mathrm{C} / \mathrm{m}, 5<\tau<50 \mu \mathrm{s}$, and $1<\mathrm{E}<4 \mathrm{MeV}$ at injection. Previous scaled heavy-ion transport experiments had much less space-charge intensity $(\lambda<0.04 \mu \mathrm{C} / \mathrm{m})$ and were not capable of resolving scale-dependant issues in transport. A goal of the HCX is to identify and understand processes that constrain choices of machine aperture needed for high confidence, but attractive, driver designs.

\section{SCIENTIFIC OBJECTIVES}

Processes listed below can limit the transportable beam current. Moreover, the deleterious effects noted can be enhanced at the high fill factors desirable for HIF. The objective of the HCX is to obtain data on how these processes limit transportable beam current.

(1) Nonlinear fields: The transverse phase space of the beam can be diluted (emittance growth) due to nonlinear fields resulting from imperfections in the applied focusing and self-field effects such as image charges and nonlinear distribution of space-charge.

(2) Alignment errors: Misalignments cause oscillations in the beam centroid with a period characterized by $\sigma_{0}$, the undepressed particle phase advance. The oscillations bring the beam-edge closer to the physical aperture, resulting in larger nonlinear forces due to applied field errors and image charges. The frequency of needed steering corrections for tolerable centroid errors is important.

(3) Distribution errors: Distribution distortions and mismatches in phase-space can launch deleterious collective oscillations. Mismatches of the distribution to focusing structures lead to beam envelope oscillations that can pump large amplitude beam halo. Higher order distortions such as those resulting from space-charge nonuniformities may launch spectrums of collective waves that complicate beam control and can lead to emittance growth. These envelope and collective modes have rapid frequency oscillations that scale with the ion plasma frequency. Broad spectrums of modes can rapidly phase mix and undergo collective relaxation with some 
emittance growth, whereas lower order modes (narrow spectrum) can persist and complicate beam control.

(4) Lost particles: Beam interactions with background gas, charge exchange, and large amplitude beam halo can result in particles lost to the machine aperture. Over a long pulse, this can result in cascades of desorbed gas being released from surfaces and production of secondary electrons that will interact with the beam pulse. At high energy, lost beam ions can also activate accelerator structures. The yield of desorbed atoms is expected to be large and has considerable impact on the needed aperture, but little relevant data exists to help fix needed beam edge to aperture clearances for characteristic HIF pulse durations and vacuum systems. Secondary electrons are also an issue of strong concern in magnetic focusing systems. Such systems lack the strong sweeping fields associated with electric focusing, increasing chances that electrons will be trapped in the multi-kV potential well of the ion-beam thereby leading to streaming instabilities, partial neutralization, and loss in beam control.

(5) Longitudinal physics: Matching of the beam head and maintaining beam control over the pulse in the presence of acceleration and velocity tilt for compression introduces various constraints. Beam-beam interactions in acceleration gaps (electric and magnetic) and focusing arrays (magnetic) may also complicate beam control.

\section{EXPERIMENTAL APPROACH}

Phase I will primarily test the physics of electrostatic quadrupole (ESQ) transport and Phase II will test magnetic quadrupole transport with one coasting, driverscale beam. To address scientific transport issues early, with limited funding, near-term plans do not include beam acceleration and compression or mutibeam arrays to study multi-beam control and beam-beam interactions. However, later stages will include at least one induction cell for the study of bunch end control and longitudinal space-charge waves. The Phase II lattice is being designed to allow retrofits for beam acceleration and compression. Some multibeam elements may be incorporated to allow mock-up of multibeam effects.

\section{Phase I}

The experimental hardware includes a $0.6 \mathrm{~A}, 1.8 \mathrm{MeV}$ $\mathrm{K}^{+}$injector and matching section $[3,4]$, followed by a 20 period FODO transport lattice of 40 ESQs (4 tanks, each with 10 ESQs), and then four pulsed magnetic quadrupoles. The ESQ lattice has a half-period of $\mathrm{L}=21.76 \mathrm{~cm}$, a clear bore radius of $\mathrm{r}_{\mathrm{p}}=2.3 \mathrm{~cm}$, and a hardedge equivalent quadrupole occupancy of $\eta \approx 0.7$ [1]. The ESQs will be aligned to within tolerances of $\pm 75 \mu \mathrm{m}$ of center, and the ESQ electrodes will be biased to $\mathrm{V} \approx$ $\pm 55 \mathrm{kV}$ at the maximum allowable focusing strength (i.e., the envelope stability limit corresponding to $\sigma_{o}=90^{\circ}$ ). The HCX ESQs are derived from a simple design of cylindrical rods mounted to end-plates, with the radii of the rods chosen to be $(8 / 7) r_{p}$ to eliminate lowest-order systematic multipole errors (dodecapole component) in $2 \mathrm{D}$ transverse geometry. The clear bore radius $r_{p}$ was set from estimates of needed beam-edge to aperture clearances including centroid errors and mismatch. Electrode gaps were set from high-voltage breakdown tests[5]. A prototype ESQ was tested to $\mathrm{V}= \pm 120 \mathrm{kV}$ (> $2 \mathrm{x}$ operating) without breakdown. A system base pressure of less than $10^{-7}$ Torr is expected.

Considerable flexibility has been designed into the lattice to allow the aperture to be probed with the beam, and effects measured. In each tank of 10 ESQs, the first ESQ will be removable to allow space for horizontal and vertical plane slit-scanners for measuring the beam phasespace in each transverse plane. This ESQ may also be rotated (up to $\pm 4^{\circ}$ ) about the reference beam centroid to introduce variable skew coupling for the study of rotational alignment tolerances. At the end of each tank, the voltages on the final four ESQs may be varied independently to adjust beam envelope excursions for matching, or purposely mismatching the rms envelope. The last two ESQs can also be displaced along the horizontal and vertical directions (over $\pm 1.2 \mathrm{~cm}$ with \pm 12 $\mu \mathrm{m}$ resolution) to allow the beam centroid to be steered in offset and angle. There will also be slit-scanner diagnostics in a tank at the end of the machine and the matching section before the first tank also has provisions for beam steering. Thus the beam centroid and envelope may be controlled and diagnostics performed near all tank to tank transitions. Initial experiments with a first tank of 10 quadrupoles are scheduled to start late in 2001.

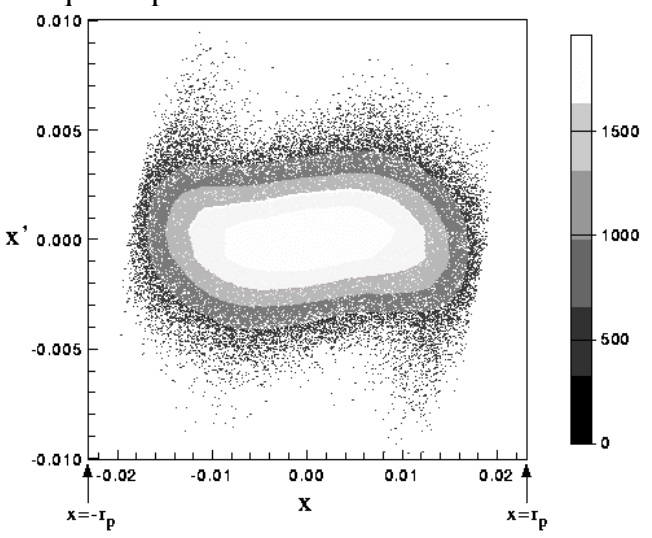

Figure 1: Transverse $x-x^{\prime}$ phase space from a 2D PIC simulation of an initially uniform (semi-Gaussian) beam transported through 5 periods of the Phase I lattice at reduced focusing strength $\left(\sigma_{0}=55^{\circ}\right)$ to fill the aperture.

An experimental program has been developed to explore emittance growth and beam control with variations in the aperture fill factor. The maximum transverse matched beam-edge excursion with zero centroid error is about $62 \%$ of the aperture at a nominal focusing strength corresponding to $\sigma_{o}=80^{\circ}$. Beam excursions in the aperture will be increased and phasespace distortions measured (emittance growth) by: lowering the focusing gradient (i.e., decreasing $\sigma_{\mathrm{o}}$ ), deliberately inducing envelope mismatch with controlled 
amplitude and phase, and by steering the beam centroid away from the nominally aligned condition. Contingent on early results, the aperture on subsequent tanks may also be changed. To probe sensitivities, the 40 quadrupole lattice will be long enough to allow $\sim 5$ undepressed and $\sim 1.2$ depressed characteristic single-particle oscillations, and $\sim 6.9$ collective plasma periods of space-charge mode evolution. A typical distribution distortion obtained for a large fill factor with an aligned beam (zero centroid error) is shown in Fig. 1.

The beam for the HCX will be produced from a retrofit ESQ injector and an ESQ matching section that were originally constructed at LBNL to deliver a $2 \mathrm{MeV}$, $800 \mathrm{~mA} \mathrm{~K}^{+}$beam for the Elise project[3]. The optics of the injector diode have been improved, the pulse length extended[5], and stable operation at $1.8 \mathrm{MeV}$ and $0.6 \mathrm{~A}$ is expected. Simulations and experiment found that various processes could result in complex distribution distortions at the exit of the injector. The retrofits have reduced these distortions, and the beam has been diagnosed extensively at the exit of the injector so that limitations imposed can be understood. If necessary, further retrofits will be carried out during the Phase I experiments.

Four pulsed magnetic quadrupoles designed for a prototype 21-beam array have been built and tested for $>80,000$ pulses[6] and will be adapted for use at the end of the Phase I lattice. The quadrupoles have an elliptical clear bore aperture with radii 3.0 and $5.0 \mathrm{~cm}$, an effective axial field length of $31 \mathrm{~cm}$, and a gradient of $40 \mathrm{~T} / \mathrm{m}$. This short lattice will provide an early, inexpensive opportunity to develop diagnostics sensitive to electrons that may become entrained in the self-field potential well of the ion beam in the absence of sweeping fields associated with ESQ focusing.

\section{Diagnostics}

Various pulse-resolved diagnostics will be available. Double-slit intercepting diagnostics similar to those employed on the ESQ injector will be employed to measure the transverse phase space (x-y, $x-x^{\prime}$ and $\left.y-y^{\prime}\right)$ of the ion beam along the horizontal ( $\mathrm{x}$ ) and vertical (y) planes. This technique relies on the demonstrated shot-toshot repeatability of such systems. The total ion linecharge density $\lambda_{\text {ion }}(t)$ (or current) will be accurately measured (1-2\%) with a beam-enclosing Faraday cup. A nearby Rogowski loop will measure net line-charge $\lambda_{\text {net }}(\mathrm{t})$ (or current). Together, these will give information on beam loss and the degree of partial beam neutralization due to secondary electrons. A valve will be present to puff gas into the vacuum system and vary the background pressure and beam-gas interactions. Halo-induced desorption will be detected with ionization gauges. A gated beam imager is being evaluated for rapid, one-shot phase-space measurements complimentary to those of the slit scanners. This system works by imaging pinholes and can provide full cross-plane correlation data on the beam distribution.
The experiment will also be employed as a test bed for new diagnostics, particularly non-intercepting and electron diagnostics. Azimuthally segmented capacitive probes will be employed to measure $\lambda_{\text {net }}(t)$ and ratios of beam envelope radii. These probes can be installed inside the bores of magnetic quadrupoles, where electrons may be trapped in orbits around the quadrupole field lines. Lowfrequency interferometry and compact energy analyzers are being evaluated to measure secondary electron effects.

\section{Phase II}

Preliminary plans call for the lattice to be extended by the addition of 50-100 magnetic quadrupoles to allow sensitive probing of magnetic transport limits, which are less understood than electric limits. A syncopated FODO lattice is envisioned with periodicity $2 \mathrm{~L}=45 \mathrm{~cm}$, clear bore radius $r_{p}=3 \mathrm{~cm}$, and a quadrupole occupancy of $\eta=0.45$. Two independent superconducting magnet designs have been successfully prototyped[7]. One of these prototypes achieved, without quench training, an integrated gradient of $8.5 \mathrm{~T}$ needed for matched beam focusing. Selfconsistent beam simulations indicated that end compensations may be employed to achieve high average field quality with simple magnet designs, thereby allowing considerable design freedom. Cryostat and lattice period designs have up to $8 \mathrm{~cm}$ free, warm axial space within the lattice period for diagnostics, pumping, acceleration gaps. Although plans call for initial coasting beam experiments, hardware is being designed to allow a later upgrade with acceleration gaps and induction cores. Pulsed magnets are also being evaluated as a cheaper, more flexible option.

\section{SUMMARY}

The HCX experiment is being built to explore intense beam transport limits at a scale typical of the low kinetic energy end of a multi-beam HIF driver. A single, coasting beam is employed for economy. The experiment will be carried out in a phased implementation with Phase I (in assembly) testing ESQ transport, and Phase II (in planing) testing magnetic quadrupole transport. Later phases may test acceleration, bunch compression, and other issues.

\section{REFERENCES}

[1] V. Karpenko et al., "An Engineering Overview of an Electrostatic Quadrupole Lattice for a High Current Transport Experiment," these proceedings.

[2] C. Celata et al., "PIC Simulations of Beam Dynamics Experiments on the HCX," these proc.

[3] S. Yu et al., "2MV Inj. as the Elise Front-End \& Exp. Facility," Fusion Eng. \& Des., 32-33 (1996) 309-315.

[4] F. Bieniosek et al., "2-MV Inj. for HCX," these proc.

[5] A.Faltens and P.Seidl, "Devel. of Elec. Quadrupoles for HIF," XVIIth Int. Symp. on Discharges and Elec. Insulation in Vac., July 1996. IEEE cat. \#96CH35939.

[6] D. Shuman et al., "A Pulsed Magnetic Quadrupole Array for HIF, ”Proc. Part. Accel. Conf. (1999) p. 785.

[7] G. Sabbi et al., "Development of Superconducting Quadrupoles for HIF," these proceedings. 